\title{
A Stabilized Fiber Laser for High-Resolution Low-Frequency Strain Sensing
}

\author{
Timothy T-Y. Lam, Jong H. Chow, Conor M. Mow-Lowry, David E. McClelland, and Ian C. M. Littler
}

\begin{abstract}
We frequency stabilize a fiber laser for use in low-frequency sensing applications. Using a radio frequency locking technique, an Erbium-doped single longitudinal mode fiber laser is stabilized to a Mach-Zehnder interferometer. The low-frequency fiber laser noise was suppressed by more than 1.5 orders of magnitude at frequencies below $300 \mathrm{~Hz}$ reaching a minimum of $2 \mathrm{~Hz} / \sqrt{\mathrm{Hz}}$ between 60 and $250 \mathrm{~Hz}$. The corresponding strain sensitivities are $2 \mathrm{p} \epsilon / \sqrt{\mathrm{Hz}}$ at $1 \mathrm{~Hz}$ and $15 \mathrm{f} \epsilon / \sqrt{\mathrm{Hz}}$ from 60 to $250 \mathrm{~Hz}$.
\end{abstract}

Index Terms-Fiber lasers, frequency locked loops, frequency stability, laser stability, laser stabilization, measurement, optical fiber sensors, strain sensing.

\section{INTRODUCTION}

$\mathbf{T}$ HE DEVELOPMENT of fiber strain sensors has attracted much interest over the past few decades [1], [2]. High-resolution strain measurements can be converted into sensitive measurements of acceleration, hydrodynamic pressure, or even magnetic field anomalies, via appropriate transducers. Fiber interferometric sensors are playing an increasingly important role in these applications. Active and passive fiber sensors have been developed to create high-performance low-frequency $(<1 \mathrm{kHz})$ strain sensing systems. Strain sensitivities recorded include 0.3 picostrain at $100 \mathrm{~Hz}$ [3] and 80 picostrain at 100 $\mathrm{mHz}$ [4]. However, for some applications in surveillance and geophysical exploration, higher sensitivities are desired.

High-resolution measurements in both active and passive sensors have been shown to be predominantly limited by laser frequency noise, with electronic noise, shot noise, and sensor thermal noise playing secondary roles [3]. A direct relation between the frequency noise and strain sensitivity of an interferometric sensor is given by [5]

$$
\frac{\Delta \nu}{\nu}=k \epsilon
$$

where $\epsilon$ is the strain applied to the sensor, $\Delta \nu$ is the frequency detuning observed from the sensor, $\nu$ is the frequency of the original frequency observed from the sensor, and the factor $\underline{k}$ is dependent on the strain optic effect in fiber and is estimated

Manuscript received December 10, 2008; revised January 30, 2009; accepted February 05, 2009. Current version published July 09, 2009. This work was supported in part by the Australian Research Council The associate editor coordinating the review of this paper and approving it for publication was Dr. Krikor Ozanyan.

The authors are with the Centre for Gravitational Physics, Australian National University, Canberra, ACT 2606, Australia (e-mail: timothy.lam@anu.edu.au).

Color versions of one or more of the figures in this paper are available online at http://ieeexplore.ieee.org.

Digital Object Identifier 10.1109/JSEN.2009.2024040 to be 0.72 for SMF-28 fiber [6]. From (1), it can be seen that to improve strain sensor resolution, frequency noise $(\Delta \nu)$ must be reduced. Distributed Bragg reflector fiber lasers are excellent sources for sensing as they have very low-frequency noise above several tens of $\mathrm{kHz}$. However, many sensing applications require sensitivity at lower frequencies. In the low-frequency regime, fiber laser frequency noise is significantly larger than at high frequencies [7], [8], thus limiting sensitivity below a few hundred $\mathrm{Hz}$. In the case of a passive sensor system, where the sensor is remotely interrogated, active frequency stabilization can be used to improve the frequency noise of the laser.

In this paper, we present a stabilized fiber laser system with very low-frequency noise for use in a variety of optic fiber point sensor systems operating in a band below $1 \mathrm{kHz}$. By tapping off some of the fiber laser output, the laser is locked, using radio frequency transmission locking, to a reference Mach-Zehnder interferometer. We provide a summary of radio frequency transmission locking and present the Mach-Zehnder interferometer as a frequency discriminator. Finally, the frequency noise of the stabilized fiber laser and corresponding strain sensitivity is demonstrated by interrogating a second Mach-Zehnder interferometer frequency discriminator.

\section{PRinciPles of RAdio FreQuency (RF) StABilization}

\section{A. Radio Frequency (RF) Transmission Locking}

Radio frequency (RF) transmission locking is a technique used to stabilize the frequency noise of a laser by locking it to a stable optical frequency reference [9]. RF transmission locking phase interrogates the frequency reference, providing a signal that is proportional to the gradient of the intensity response. This method is immune to small intensity fluctuations of the laser [10]. To interrogate the sensor, phase modulation of the laser carrier frequency is used to create sidebands that co-propagate with the laser beam. Beating between the sidebands and carrier frequency after interaction with the phase response of the optical reference produces amplitude modulation that is proportional to the frequency detuning between the laser and frequency reference. The amplitude modulated signal is then demodulated, generating a DC signal which may be used to feed back to a frequency actuator to suppress frequency fluctuations of the laser. In principle, the laser noise may be suppressed to the shot noise limit. However, in most physical systems this limit is rarely achieved due to other noise sources, such as electronic or acousto-mechanical noise coupling into the optical frequency reference. Any noise experienced by the optical reference is imposed on the laser. Appropriate choice of an optical reference, as well as adequate mechanical isolation, is required to minimize external noise coupling. 


\section{B. The Mach-Zehnder Frequency Discriminator}

An arm length mismatched Mach-Zehnder interferometer is a two beam interference frequency discriminator. The output intensity varies sinusoidally with input frequency, allowing for many possible lock points. A fiber Mach-Zehnder is an easily adjustable, small footprint, and all fiber reference with operating wavelengths extending over the range of operation of the input and output couplers. The amplitude transmission coefficient of the Mach-Zehnder interferometer is

$$
F(\nu)=1+\exp \left(i \frac{2 \pi n \nu \Delta L}{c}\right)
$$

where $n$ is the refractive index of the fiber $(\sim 1.5), c$ is the speed of light, and $\Delta L$ is the arm length mismatch. The intensity response of the Mach-Zehnder is then

$$
I_{\mathrm{out}}=\frac{I_{\mathrm{inc}}}{2}\left(1+\cos \left(\frac{2 \pi n \nu \Delta L}{c}\right)\right)
$$

where $I_{\text {inc }}$ is the intensity of the input beam. Note that the response has a DC offset that is proportional to the intensity of the input. By phase modulating the input beam and demodulating the output beam this DC offset is be removed. The transmission of the phase modulated beam through an interferometer is given by $[11]$

$$
\begin{aligned}
T_{m} \propto \operatorname{Re} & \left\{F(\nu) \tilde{F}\left(\nu_{+}\right)-\tilde{F}(\nu) F\left(\nu_{-}\right)\right\} \cos \left(2 \pi \nu_{m} t\right) \\
& +\operatorname{Im}\left\{F(\nu) \tilde{F}\left(\nu_{+}\right)-\tilde{F}(\nu) F\left(\nu_{-}\right)\right\} \sin \left(2 \pi \nu_{m} t\right)
\end{aligned}
$$

where $\nu_{+}=\nu+\nu_{m}, \nu_{-}=\nu-\nu_{m}$, and $\nu_{m}$ is the modulation frequency. Mixing this signal with the modulation frequency will produce a DC term and some higher order terms that are removed using a low-pass filter. Recalling the Mach-Zehnder transmission in (2), we obtain

$$
\begin{aligned}
\varepsilon \propto \frac{1}{2}[\operatorname{Re} & \left\{\left(1-\exp \left(\frac{-i 2 \pi \Delta L \nu}{c}\right)\right)\right. \\
& \left.\times\left(i \sin \left(\frac{2 \pi \Delta L \nu}{c}\right)\right)\right\} \cos (\Psi) \\
& +\operatorname{Im}\left\{\left(1-\exp \left(\frac{-i 2 \pi \Delta L \nu}{c}\right)\right)\right. \\
& \left.\left.\times\left(i \sin \left(\frac{2 \pi \Delta L \nu}{c}\right)\right)\right\} \sin (\Psi)\right]
\end{aligned}
$$

where $\varepsilon$ is the demodulated error signal and $\Psi$ is the phase difference between the demodulation signal and the modulation signal. The error signal contains information in both the real and imaginary quadratures. By choosing the correct phase, one quadrature is selected. The resultant signal, which is a function of both frequency and displacement, has no DC offset and provides a zero crossing at either a maximum or a minimum of the interferometer output. For frequencies close to a zero crossing, the signal is linearly proportional to frequency and displacement fluctuations.

The steepness of the frequency discriminator provided by a Mach-Zehnder depends on the fringe spacing, or the frequency

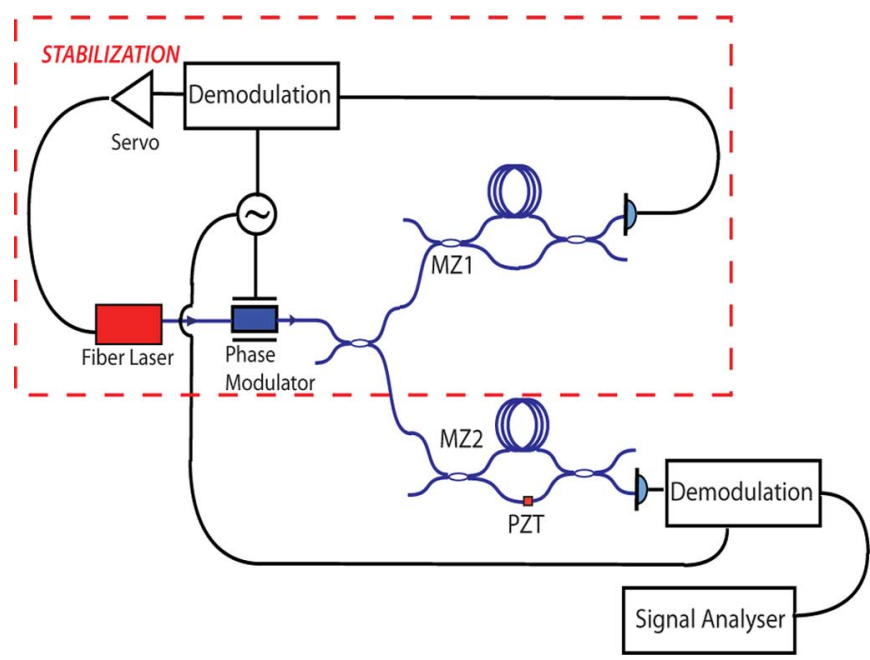

Fig. 1. A schematic illustration of the experiment. A 50:50 coupler is used to split the beam between the stabilization and sensing paths. The modulation and demodulation is driven by a single $60 \mathrm{MHz}$ signal generator. A PZT was attached to one arm of the measurement interferometer (MZ2) to allow independent control of its interference condition.

separation of adjacent maxima. Equation (3) can be easily manipulated to produce

$$
\Delta \nu_{\text {fringe }}=\frac{c}{n \Delta L}
$$

where $\Delta \nu_{\text {fringe }}$ is the fringe spacing of the interferometer. From (6), it can be seen that an increase in arm length mismatch will decrease the fringe spacing. Decreasing the fringe spacing increases the gain the of the frequency discriminator, producing a larger response for a given perturbation.

\section{EXPERIMENTAL SETUP}

The experimental setup is shown in Fig. 1. The output of a narrow linewidth fiber laser (Orbits Lightwave Ethernal) was split into two beams using a 50:50 coupler. One beam was used to lock the laser to a reference Mach-Zehnder interferometer (MZ1) and the other was used to interrogate a measurement interferometer (MZ2). Both interferometers were located in a single isolation chamber for maximum common noise rejection. The Mach-Zehnder interferometers were constructed using two 50:50 couplers spliced together with an additional length of SMF-28 fiber in one arm giving the arm length mismatch.

\section{A. Stabilization}

The laser was locked, using RF transmission locking, to a dark fringe of MZ1. MZ1 had a 20 meter arm mismatch, providing a $10 \mathrm{MHz}$ fringe spacing. To reduce thermal fluctuations and external noise coupling, the interferometer arms were wound around a metal spool and placed in a dense walled, acoustically damped isolation chamber, which was mounted on damped isolating footings. An electro-optic modulator (JDSU CATV Band Dual Output Modulator) was used to introduce $60 \mathrm{MHz}$ phase modulation sidebands. The modulated beam was used to phase interrogate MZ1. The transmission of the interferometer was detected using a RF photodetector and the signal demodulated via a mixer and low pass filter to obtain an error signal. This error signal was filtered and amplified using 


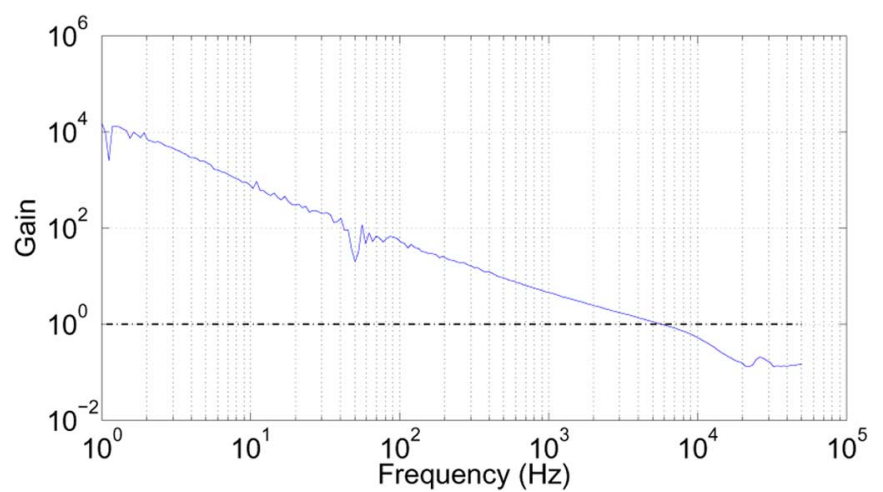

Fig. 2. The open loop gain of the stabilization system. The plot shows a $1 / f$ gain roll-off from low frequencies with a gain of 57 at $100 \mathrm{~Hz}$. The system's unity gain frequency is $5.7 \mathrm{kHz}$.

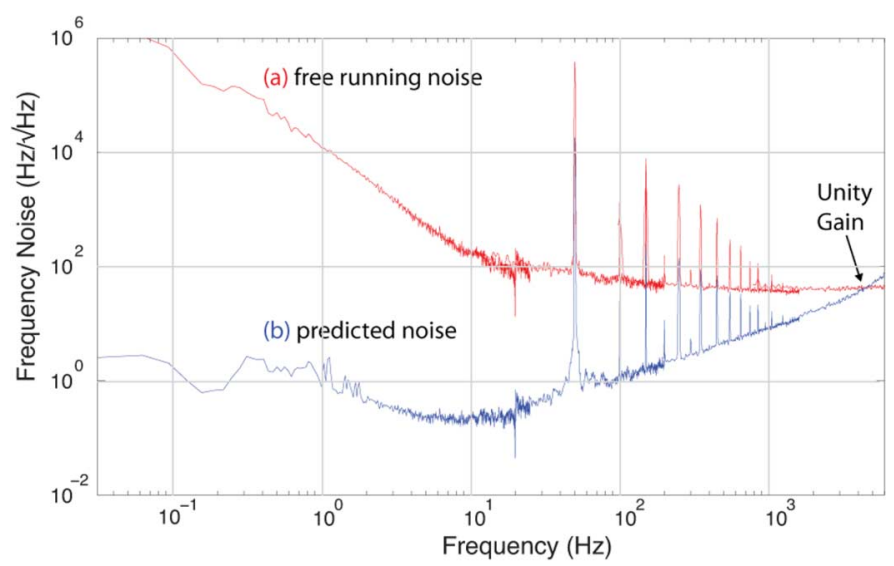

Fig. 3. The predicted suppression of the laser frequency noise. (a) Shows the free running laser noise. (b) Shows the simulated suppressed frequency noise. A suppression of $20 \mathrm{~dB}$ is seen at $10 \mathrm{~Hz}$ with unity gain reached at $5.7 \mathrm{kHz}$. Resonant peaks at $50 \mathrm{~Hz}$ and its harmonics are due to electronic mains coupling into the system.

a frequency servo and is fed back to the laser piezo-electric transducer (PZT) to tune the laser frequency.

The response of the frequency servo was designed to provide as high a bandwidth as possible given the mechanical response of the PZT frequency actuator. Fig. 2 shows the open loop gain of the stabilization system. The open loop transfer function had a unity gain of $5.7 \mathrm{kHz}$. A $10 \mathrm{~Hz}$ passive filter was used to provide a " $1 / f$ " roll-off from low frequencies. When lock was acquired, an active integrator was engaged to further suppress low-frequency fluctuations and prevent drift. This provided a gain of 57 at $100 \mathrm{~Hz}$. The simulated stabilized frequency noise is shown in Fig. 3. A suppression of more than two orders of magnitude is predicted at $10 \mathrm{~Hz}$ with frequency noise reaching $0.3 \mathrm{~Hz} / \sqrt{\mathrm{Hz}}$. Large resonant peaks at $50 \mathrm{~Hz}$ and its harmonics are due to electronics mains coupling into the system.

\section{B. Noise Measurement}

The stabilized fiber laser output was used to interrogate MZ2. MZ2 is a Mach-Zehnder interferometer with a $40 \mathrm{~m}$ arm length mismatch. The light transmitted through the sensor was detected on a photodetector and demodulated to produce an error signal, as with MZ1. The error signal was sent to an audio-band spectrum analyzer where an fast Fourier transform (FFT) of the signal was performed.
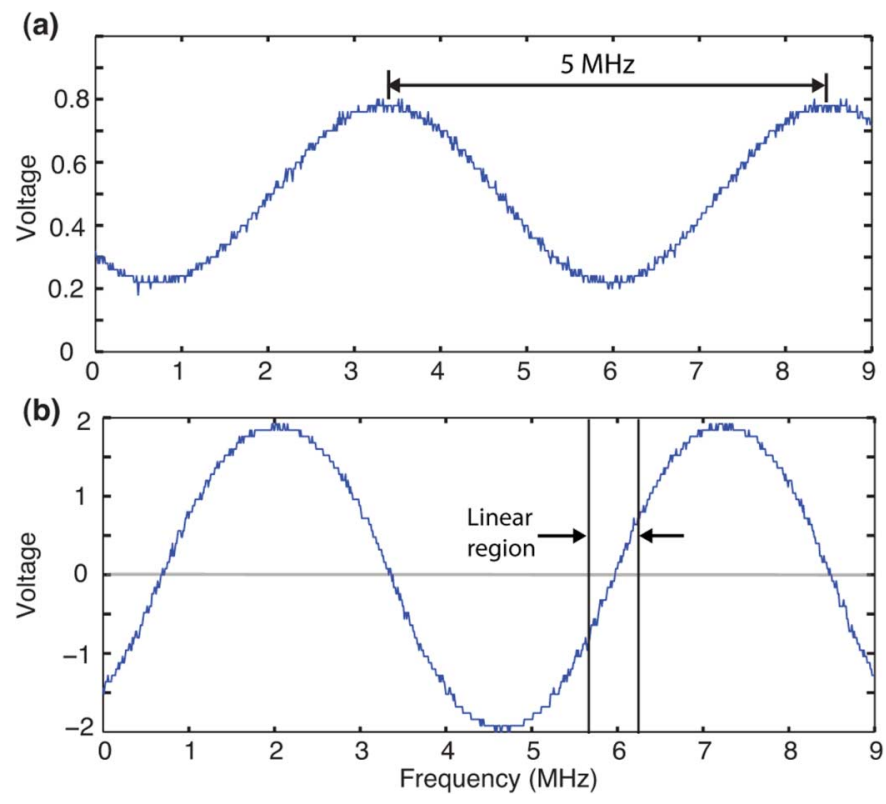

Fig. 4. The transmission (a) of the Mach-Zehnder sensor (MZ2) and its corresponding error signal (b) scaled to frequency. The error signal has a peak to peak voltage of $3.9 \mathrm{~V}$. The slope in the linear region is $2.4 \times 10^{6} \mathrm{~V} / \mathrm{Hz}$. Note that the transmission minima and maxima correspond to zero crossings on the error signal.

Fig. 4 shows (a) the transmission of interferometer MZ2 and (b) its corresponding error signal when the frequency of the laser is scanned. The error signal had a peak to peak voltage of 3.9 V. From Fig. 4, it can be seen that the error signal has no DC offset and that the zero crossings of the error signal correspond to the maxima and minima of the transmission. To perform an out-of-loop measurement of the laser noise using MZ2, the interferometer was tuned to the linear region of the error signal by applying a DC offset to a PZT which stretched one arm of the interferometer. In the linear region, the MZ2 error signal voltage varies linearly with frequency detuning. The measurement was calibrated with the knowledge that one fringe space is the equivalent of $5 \mathrm{MHz}$. Thus, the slope of the error signal in the linear region is $2.4 \times 10^{6} \mathrm{~V} / \mathrm{Hz}$. The noise spectrum generated by the signal analyzer was scaled by the slope of the error signal to obtain a power spectrum of the frequency noise of the system.

The electronic noise of the system was measured by disconnecting the optical input to the photodetectors and measuring the noise at the output of the demodulation electronics. This was then added in quadrature to the calculated voltage equivalent shot noise of the system given by [12]

$$
V_{\mathrm{SN}}=\alpha \sqrt{2 e g V_{\mathrm{DC}}}
$$

where $V_{\mathrm{DC}}$ is the $\mathrm{DC}$ voltage on the photodetector, $\alpha$ is the gain, $e$ is $1.602 \times 10^{-19}$ Coulombs, and $g$ is the transimpedance gain of the photodetector. The shot noise voltage is then converted into strain using the error signal slope and arm length.

In order to keep the strain sensor in the linear region of the error signal, a low bandwidth control system was implemented. This control loop was designed with a unity gain of $10 \mathrm{~Hz}$ with a $1 / f$ roll-off from DC. The sensor spectrum within the bandwidth of the controller was divided by the measured suppression of this control loop to determine the actual sensitivity observed. 


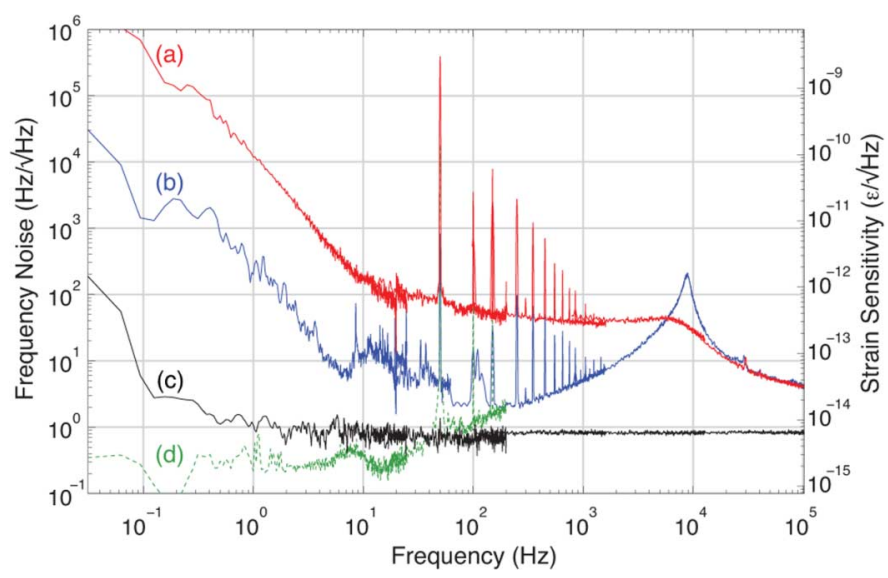

Fig. 5. The strain sensitivity as obtained by the Mach-Zehnder strain sensor. The plot showed (a) the free running strain performance of the laser, (b) the stabilized performance of the laser, (c) the quadrature sum of the shot noise and the photodetector and mixer electronic noise of the sensor, and (d) predicted performance of the stabilized laser. Peaks at $50 \mathrm{~Hz}$ and its multiples are due to mains coupling. At $250 \mathrm{~Hz}$, the open system diverges from the predicted performance and is limited by seismic roll up and an unknown noise floor slightly above the electronic noise.

\section{RESULTS}

Fig. 5 shows the frequency noise (left vertical axis) and strain sensitivity (right vertical axis). Active stabilization of the laser reduced the free running noise below $5.7 \mathrm{kHz}$. The free running frequency noise was suppressed by more than 1.5 orders of magnitude, reducing the noise from $60 \mathrm{~Hz} / \sqrt{\mathrm{Hz}}$ to $2 \mathrm{~Hz} / \sqrt{\mathrm{Hz}}$ between 70 and $250 \mathrm{~Hz}$. This noise increases to $200 \mathrm{~Hz} / \sqrt{\mathrm{Hz}}$ at $1 \mathrm{~Hz}$. Below $250 \mathrm{~Hz}$ the stabilized sensitivity diverges from the predicted performance and hits a noise floor slightly above the combination of shot noise and electronic noise. Noise roll up below $5 \mathrm{~Hz}$, as well as the broad peaks between 8 and $33 \mathrm{~Hz}$, are attributed to mechanical noise in the Mach-Zehnder interferometers. Large peaks at $50 \mathrm{~Hz}$ and its harmonics are caused by the mains frequency coupling into the system. The large peak at $8 \mathrm{kHz}$ is due to noise amplification near the unity gain of the servo.

A direct measurement of the strain sensitivity was made by calibrating MZ2 to an equivalent strain sensitivity measurement. This calibration was made by knowing that a displacement of one wavelength will produce a change of one fringe. With this calibration, sensitivities better than $100 \mathrm{f} \epsilon / \sqrt{\mathrm{Hz}}$ were recorded between $3 \mathrm{~Hz}$ and $3 \mathrm{kHz}$. The system reached its highest sensitivity between 70 and $250 \mathrm{~Hz}$ where the free running laser sensitivity was reduced from an equivalent strain of $300 \mathrm{f} \epsilon / \sqrt{\mathrm{Hz}}$ to a stabilized sensitivity of $15 \mathrm{f} \epsilon / \sqrt{\mathrm{Hz}}$. At $100 \mathrm{mHz}$, a strain of $10 \mathrm{p} \epsilon / \sqrt{\mathrm{Hz}}$ was measured. This falls to $2 \mathrm{p} \epsilon / \sqrt{\mathrm{Hz}}$ at $1 \mathrm{~Hz}$. Frequency stabilization of the laser provides a $30 \mathrm{~dB}$ improvement in acoustic and quasi-static strain sensitivity.

\section{CONCLUSION}

We have demonstrated an actively stabilized laser with considerably improved frequency noise performance from $5.7 \mathrm{kHz}$ to infrasonic and quasi-static frequencies of several $\mathrm{mHz}$, when compared with a free running fiber laser. A minimum of $2 \mathrm{~Hz} / \sqrt{\mathrm{Hz}}$ was achieved between 60 and $250 \mathrm{~Hz}$ with the frequency noise rolling up to $200 \mathrm{~Hz} / \sqrt{\mathrm{Hz}}$ at $1 \mathrm{~Hz}$. The stabilized laser can be used to interrogate various types of passive strain sensors for a range of applications. Equivalent strain sensitivities of the laser were $15 \mathrm{f} \epsilon / \sqrt{\mathrm{Hz}}$ between 60 and $250 \mathrm{~Hz}$. This shows an improvement of $30 \mathrm{~dB}$ over the sensitivity of a free running fiber laser.

\section{REFERENCES}

[1] A. D. Kersey, "A review of recent developments in fiber optic sensor technology," Opt. Fiber Technol., vol. 2, no. 36, pp. 291-317, 1996.

[2] B. Lee, "Review of the present status of optical fiber sensors," Opt. Fiber Technol., vol. 9, no. 2, pp. 57-79, 2003.

[3] J. H. Chow, I. C. M. Littler, D. McClelland, and M. Gray, "Demonstration of a passive sub-picostrain fiber strain sensor," Opt. Lett., vol. 30, pp. 1923-1925, 2005.

[4] N. Beverini, E. Maccioni, M. Morganti, F. Stefani, R. Falciai, and C. Trono, "Fiber laser strain sensor device," J. Opt. A: Pure Appl. Opt., vol. 9, pp. 958-962, 2007.

[5] G. A. Cranch, G. M. H. Flockhart, and C. K. Kirkendall, "Distributed feedback fiber laser strain sensors," IEEE Sensors J., vol. 8, pp. 1161-1172, 2008.

[6] C. Butter and G. Hocker, "Fiber optic strain gauge," Appl. Opt., vol. 17, pp. 2867-2869, 1978.

[7] G. A. Ball, C. Huli-Allen, and J. Livas, "Frequency noise of a Bragg grating fibre laser," Electron. Lett., vol. 30, pp. 1229-1230, 1994.

[8] C. Spiegelberg, J. Geng, Y. Hu, Y. Kaneda, S. Jiang, and N. Peyghambarian, "Low-noise narrow-linewidth fiber laser at $1550 \mathrm{~nm}$ (june 2003)," J. Lightwave Technol., vol. 22, pp. 1229-1230, 2004.

[9] A. D. White, "Frequency stabilization of gas lasers," IEEE J. Q. Elect., vol. 1, p. 349, 1965.

[10] E. Bava, G. Galzerano, and C. Svelto, "Amplitude and frequency noise sensitivities of optical frequency discriminators based on fabry-perot interferometers and the frequency modulation technique," Rev. Sci. Instrum., vol. 77 , no. 12 , pp. 123106-1, 2006.

[11] E. Black, "An introduction to pound-drever-hall laser frequency stabilization," Amer. J. Phys., vol. 69, no. 1, p. 79, 2001.

[12] A. Yariv, Quantum Electronics, 3rd ed. New York: Wiley, 1989.

Timothy T-Y. Lam received the B.E. degree from the Australian National University, Canberra, in 2007. He is currently working towards the Ph.D. degree at the Australian National University.

His research interests include fiber-based and free-space optical sensors.

Jong H. Chow is a Postdoctoral Fellow at the Australian National University, Canberra.

His research interests include free-space and fiber-based optical sensors.

Conor M. Mow-Lowry is currently working towards the Ph.D. degree at the Australian National University, Canberra.

He is a Research Associate at the Centre for Gravitational Physics, Australian National University.

David E. McClelland received the Ph.D. degree from the University of Otago (UO), Otago, New Zealand, in 1987.

He was awarded a Beverly Research Fellowship in Physics at UO before being appointed to the Australian National University (ANU), Canberra, as a Lecturer in 1988. He is now a Professor and Director of the Centre for Gravitational Physics, ANU. His research interests include the development of optical techniques for second-generation laser-interferometer-based gravitational wave detectors, the generation and application of squeezed states of light, and gravitational-wave data analysis.

Ian C. M. Littler received the Ph.D. degree (summa cum laude) from the University of Kaiserslautern, Germany, in 1991 and the M.B.A. degree from the Australian Graduate School of Management, Canberra, in 1997.

His career has been split between research in fundamental and applied science, coupled with the commercialization of technology. He has held leadership positions in technology startups, yet remains hands-on in the laboratory, publishing widely on aspects of fiber-optic technology. His current focus is on novel fiber-optic sensors and signal extraction techniques for geophysical survey, as well as the commercialization of these technologies. 